\title{
An Information Theoretic Approach for Systems with Parallel Distributions: Case Studying Internet Traffic
}

\author{
Charalabos Skianis ${ }^{1,2}$ and Lambros Sarakis ${ }^{2}$ \\ ${ }^{1}$ University of the Aegean, \\ Department of Information and Communication Systems Engineering, \\ GR-83200, Karlovassi, Greece \\ 2 National Centre for Scientific Research 'Demokritos', \\ Institute of Informatics \& Telecommunications, \\ 15310 Aghia Paraskevi Attikis, POB 60228, Athens, Greece \\ \{skianis, sarakis\}@it.demokritos.gr
}

\begin{abstract}
The principle of Minimum Relative Entropy (MRE) is applied to characterize a 'proportionality' relationship between the state probabilities of infinite and finite capacity queues at equilibrium and thus, establish an information theoretic interpretation for the exact global balance solution of some finite capacity queues with or without correlated arrival processes. This result serves to establish the utility of the MRE inference technique and encourage its applicability to the analysis of more complex, and thus more realistic, queuing systems. The principles of Maximum Entropy (ME) and MRE are then employed, as least-biased methods of inference, towards the analysis of a Internet link carrying realistic TCP traffic, that exhibit this 'proportionality' relationship between a finite and infinite buffer system, as produced by a large number of connections. The analytic approximations are validated against exhaustive simulation experiments. Despite its simplicity, the methodology captures the behavior of the system under study both in the cases of finite and infinite buffers and finally and can easily be utilized for network management and design, capacity planning, and congestion control.
\end{abstract}

\section{Introduction}

Nowadays Internet traffic is carried by networks using primarily TCP as the transport protocol. Research efforts in the field span from direct measurements and TCP protocol simulations to analytical modeling towards more elaborate problems such as network management and design, capacity planning and congestion control. In this paper, we present a simple information theoretic approach to the case of both uncongested and congested links. The specific topic is extensively studied with many efforts devoted to study characteristics of Internet traffic such as long range dependence, self-similarity, and multi-fractal scaling (e.g. [1], [2], [3]). In certain cases, traditional approaches proved inadequate to handle the complexity of the packet arrival process with new theoretical tools suggested in [4] for traffic engineering purposes. Packetlevel characteristics of Internet traffic is studied in [5], [6], [7], [8] and finite size 
TCP connections studied in [9], [10], [11]. In [12] the notion of batch arrivals is used for the study of link fed with a varying (large) number of finite TCP flows. The approach although close to simulation results under a set of assumptions proves somehow elaborate and time consuming with the calculation of the batch size distribution and the use of iterative processes in the finite buffer cases. In a similar fashion [13] considers short TCP flows that never leave slow-start and uses a burst-level M/G/1 model to evaluate the queue length distribution at the bottleneck router for the infinite buffer case. To our knowledge there is no simple and robust suggestion towards the estimation of the queue length distribution of an Internet link (with finite or infinite buffer) carrying realistic traffic as produced by converging TCP flows.

Classical queuing theory provides a conventional and powerful framework for formulating and solving models of discrete flow systems such computer systems, communication networks and flexible manufacturing systems. However, in many cases, simplified assumptions are employed in order to produce tractable solutions, whilst approximate methods are required to analyze more complex, and thus, more realistic models. Since mid-60s, alternative ideas and tools have been proposed in the literature ([14], [15], [16]).

It can be argued that one of the most fundamental requirements for the analysis of complex queuing systems is the provision of a convincing interpretation for a probability assignment free from arbitrary assumptions. In a more general context, this was the motivation behind the principles of Maximum Entropy (ME) ([17], [18]) and Minimum Relative Entropy (MRE) ([19], [20]). These principles provide selfconsistent methods of inference for estimating uniquely and in a least biased fashion an unknown but true probability distribution, based on information expressed in terms of known to exist prior distribution estimate and/or true mean value constraints. Over the recent years, these principles have been applied to characterize useful information theoretic approximations of performance distributions for queuing systems and networks and the formation of experimental performance bounds (e.g., [21], [22], [23]).

In this paper, the principle of MRE is used to determine a 'proportionality' relationship between the state probabilities of some infinite and finite capacity queues and thus, establish an information theoretic interpretation of global balance solutions for finite capacity queues with or without correlated arrival processes. The MRE methodology can be further applied to characterize exact and approximate relationships for more complex queuing systems for which the 'proportionality' relationship may not have as yet been established via classical queuing theory. Furthermore, the MRE principle may lead to cost-effective closed form solutions and performance bounds for finite capacity queues which in turn can be used as cost-effective building blocks for the performance analysis of Queueing Network Models (QNMs) of discrete-flow systems. An example of such application can be seen in [23].

The main contribution of this paper can be summarized as follows: (i) the principle of MRE is used to determine a 'proportionality' relationship between the state probabilities of some infinite and finite capacity queues and thus, establish an information theoretic interpretation of global balance solutions for finite capacity queues; (ii) we focus on an Internet link with traffic resulting out of converging flows of TCP connections and show that a 'proportionality' relationship exists between finite and infinite capacity systems; (iii) we introduce the information theoretic concepts of ME and MRE, as least-biased methods of inference, towards the estimation of the queue 
length distribution, for both finite and infinite buffer systems, based on a small set of system characteristics; (iv) we show the good fit of our approach.

The principles of ME and MRE, a generalization, are described in Section 2 whilst Sections 3 and 4 present the analysis of infinite and finite systems based on the ME and MRE principles and discuss on the 'proportionality' relationship amongst finite and infinite systems. Section 5 outlines the system setting and presents the analysis of the infinite and finite systems based on the ME and MRE principles, respectively. Extensive simulation study with ns-2 proves the good fit of the approach and the 'proportionality' relationship between internet links with finite and infinite capacity buffers. Section 6 concludes the work and suggests future directions.

\section{The Principles of ME and MRE}

Let $x$ be the state of a system with a set $D$ of feasible states. Let $D^{*}$ be the set of all the probability density functions (pdf) $p$ on $D$ such that $p(x) \geq 0, \forall x \in D$, and

$$
\int_{D} p(x) d x=1
$$

Suppose that the system under consideration is described by a true but unknown density function $p^{*} \in D^{*}$ and that $q \in D^{*}$ is a prior density that is a current estimate of $p^{*}$, such that $q(x) \geq 0, \forall x \in D$.

In addition, new information for the system places a number of constraints on $p^{*}$, in the form of expectations defined on a set of $k$ suitable functions $\left\{\alpha_{i}(x)\right\}, i=1,2, \ldots, k$ with known values $\left\{\left\langle\alpha_{i}\right\rangle\right\}, i=1,2, \ldots, k$ namely

$$
\int_{D} a_{i}(x) p(x) d x=<a_{i}>, i=1,2, \ldots, k,
$$

where $k$ is less than the number of possible states.

Since the above set of constraints (1)-(2), denoted by $I=\left(p^{*} \in \Phi\right)$ do not determine the form of $p^{*}(x)$ completely, they are satisfied by a set of pdfs $\Phi \subseteq D^{*}$.

The principle of MRE (e.g., [20]) states that of all pdfs that satisfy constraints $I$, the least biased one is the posterior pdf, $p \in \Phi$ that minimizes the relative entropy function, $H(p, q)$ in the set $\Phi$, namely

$$
H(p, q)=\min _{p^{\prime} \in \Phi} H\left(p^{\prime}, q\right)
$$

where

$$
H\left(p^{\prime}, q\right)=\int_{D} p^{\prime}(x) \log \left(p^{\prime}(x) / q(x)\right) d x .
$$

By applying the Lagrange's method of undetermined multipliers the form of the posterior pdf is [20]

$$
p(x)=q(x) \exp \left(-\beta_{0}-\sum_{i=1}^{k} \beta_{0} a_{i}(x)\right),
$$

where $\left\{\beta_{0}\right\}$ and $\left\{\beta_{i}\right\}, i=1,2, \ldots, k$ are the Lagrangian multipliers whose values are determined by the constraints (1) and (2). 
From (1) the normalizing constant is given by

$$
\exp \left(\beta_{0}\right)=\int_{D} q(x) \exp \left(-\sum_{i=1}^{k} \beta_{i} a_{i}(x)\right) d x
$$

If the integral in (6) is solved analytically, closed form expressions could be derived for $\left\{\beta_{i}\right\}, i=1,2, \ldots, k$ in terms of the mean values $<\alpha_{i}>$.

In an information theoretic context (c.f.,[17]), the ME solution corresponds to the maximum disorder of system states, and thus is considered to be the least biased distribution estimate of all solutions that satisfy the system's constraints. In sampling terms, Jaynes in [18] has shown that, given the imposed constraints, the ME solution can be experimentally realized in overwhelmingly more ways than any other distribution. Major discrepancies between the ME distribution and the experimentally observed distribution indicate that important physical constraints have been overlooked. Similar justifications can be advanced for relative entropy minimization.

In formal terms, the relative entropy minimization procedure may be seen as an information operator " $O$ " that takes two arguments, a prior distribution $q$ and a new constraint information $I$ of the form (1) and (2), yielding a posterior MRE distribution $p$, i.e. $p=q o I$. To this end it can be shown that minimization of $\mathrm{H}(p, q)$ uniquely characterizes distribution $p$, satisfying four consistency inference criteria proposed by Shore and Johnson in [20]. In particular, it has been shown that ME and MRE solutions are uniquely correct distributions and that any other functional used to implement operator " $O$ " will produce the same distribution as the entropy and relative entropy functionals, otherwise it will be in conflict with the consistency criteria.

In the field of systems modeling, expected values of various performance distributions of interest, such as the number of jobs in each resource queue concerned, are often known, or may be explicitly derived, in terms of moments of interarrival and service time distributions. Note that the determination of the distributions themselves, via classical queuing theory, may prove an unfeasible task even for systems of queues with moderate complexity. However, prior estimates of distributions may be obtained by using properties of the system, as appropriate. Hence, it is implied that the methods of entropy maximization and relative entropy minimization may be applied to characterize useful information theoretic approximations of performance distributions of queuing systems and networks.

\section{Maximum Entropy Solution of the $M^{[X]} / G / 1$ Queue}

The $\mathrm{M}^{[\mathrm{x}]} / \mathrm{G} / 1$ queues denote, single server queuing systems at equilibrium with infinite capacity, general $(\mathrm{G})$ service times and exponential $(\mathrm{M})$ batch interarrival times, respectively, where $\mathrm{x}$ is an independent random variable representing the number of customers within an arriving batch. Let at any given time, $n, n=0,1, \ldots$, be the number of packets in the system and $\{q(n)\}$ be the true but unknown steady state probability of having $n$ packets. Suppose all that is known about the state probabilities $q(n)$, $n=0,1, \ldots$, is the following set of mean value constraints: 
(i ) Normalization $\sum_{n=0}^{\infty} q(n)=1$,

(ii ) Probability of a busy system $\sum_{n=0}^{\infty} h(n) q(n)=1-q(0)$,

where $h(n)=0, n=0$, or 1 , otherwise,

( iii ) Average queue length, $<n>, \sum_{n=0}^{\infty} n q(n)=<n>$.

The probability distribution $q(n)$ can be completely specified by maximizing the entropy functions

$$
H(q(n))=-\sum_{n=0}^{\infty} q(n) \log (q(n)
$$

subject to prior information expressed in the form of constraints (7)-(9). By applying the method of Lagrange's undetermined multipliers the ME solution is expressed as

$$
q(n)=(1 / Z) g^{h(n)} x^{n}, n=0,1, \ldots,
$$

where $Z, g, x$, can be determined form the set of constraints (7)-(9).

To this end, the Maximum Entropy solution for $q(n)$ is given by

$$
q(n)= \begin{cases}1 / Z, & n=0, \\ (1 / Z) g x^{n}, & n>0,\end{cases}
$$

where

$$
1 / Z=q(0), x=1-(1-q(0)) /<n>, g=(1-q(0))(1-x) /(q(0) \cdot x)
$$

\section{Minimum Relative Entropy Solution of the $M^{[X]} / G / 1 / N$ Queue}

The $\mathrm{M}^{[\mathrm{x}]} / \mathrm{G} / 1 / \mathrm{N}$ queue denote, single server queuing systems at equilibrium with finite capacity, general (G) service times and exponential (M) batch interarrival times, respectively, where $\mathrm{x}$ is an independent random variable representing the number of customers within an arriving batch. It is assumed that all those customers that upon arrival find the buffer full are turned away. At any given time, let $n, n=0,1, \ldots, N$, be a system state representing the number of customers present in the system and $\left\{\lambda, \mathrm{Ca}^{2}\right\}$ and $\left\{\mu, \mathrm{Cs}^{2}\right\}$ the mean rate and square coefficient of variation (SCV) of the interarrival and service time distributions, respectively. Suppose that $p_{N}(n), \mathrm{n}=0,1, \ldots, \mathrm{N}$, is the true but unknown probability distribution that there are $\mathrm{n}$ customers in the system and $\mathrm{q}(\mathrm{n})$ is a prior estimate of $p_{N}(n)$. Moreover, new information takes the form of the following constraints,

(i ) The Normalization, $\sum_{n=0}^{N} p_{N}(n)=1$,

(ii) The Full Buffer State Probability, $p_{N}(N)=\varphi, 0<\varphi<1$, written as

$$
\sum_{n=0}^{N} f(n) p_{N}(n)=\varphi, f(n)=\left\{\begin{array}{ll}
0, & n<N \\
1, & n=N
\end{array},\right.
$$

and satisfying the flow balance condition

$$
\lambda(1-\pi)=\mu U,
$$

where, $\pi$ is the probability that an individual customer will be blocked upon arrival, and $\mathrm{U}$ is the utilization. 
The form of the MRE solution $p_{N}(n), \mathrm{n}=0,1, \ldots, \mathrm{N}$ can be characterized by minimizing the relative entropy function, $H\left(p_{N}, q\right)$, subject to constraints (i) and (ii). This can be achieved by applying the method of Lagrange's undetermined multipliers, leading to the MRE solution

$$
p_{N}(n)=(1 / Z) q(n) y^{f(n)},
$$

where $\mathrm{Z}$ is the normalizing constant and $\mathrm{y}$ is the Lagrangian coefficient corresponding to constraint (ii).

\subsection{The Proportionality Relationship}

For the queuing systems under consideration the stationary distribution, $p_{N}(n)$, satisfies the following set of equations

$$
p_{N}(n)=p_{N}(0) \alpha(n)+\sum_{j=1}^{n+1} p_{N}(j) \alpha(n-j+1), 0 \leq n \leq N-2,
$$

where $\alpha(n)$ is the probability that $n$ messages arrive during a service period. Note that eqs. (18) are of the same form as those for the queue length distribution (qld) $\left\{p_{\infty}(n) ; 0 \leq n \leq N-2\right\}$ of the corresponding infinite queuing system, namely

$$
p_{\infty}(n)=p_{\infty}(0) \alpha(n)+\sum_{j=1}^{n+1} p_{\infty}(j) \alpha(n-j+1), 0 \leq n \leq N-2 .
$$

By dividing the system of eqs. (18) and (19) by $p_{N}(0)$ and $p_{\infty}(0)$, respectively, ratios $p_{N}(n) / p_{N}(0)$ and $p_{\infty}(n) / p_{\infty}(0)$ become identical for $0 \leq n \leq N-1$. Thus:

$$
p_{N}(n)=\left(p_{N}(0) / p_{\infty}(0)\right) p_{\infty}(n), 0 \leq n \leq N-1 \text {. }
$$

\subsection{MRE Solution and the Proportionality Relationship}

The form of the MRE solution suggests the following proportionality relationships between the unknown probability distribution $p_{N}(n)$ and the prior estimate for that distribution, $q(n)$, namely

$$
p_{N}(n)=\left\{\begin{array}{cc}
(1 / Z) q(n), & 0 \leq n \leq N-1, \\
(1 / Z) q(n) y, & n=N .
\end{array}\right.
$$

The prior distribution estimate, $q(n)$ can be determined by the qld of the corresponding infinite capacity $\mathrm{M}^{[\mathrm{x}]} / \mathrm{G} / 1$ queues, given in Section 3. The MRE solution for the finite capacity systems, after some mathematical manipulations, can be clearly expressed by proportionality relationship (20) for $0 \leq n \leq N-1$, whilst

$$
p_{N}(n)=\left(p_{N}(0) / p_{\infty}(0)\right) p_{\infty}(n) y, n=N .
$$

The remaining unknown y can be evaluated by using the set of constraints expressed by eqs. (14) and (15) and the flow balance equation (16). Thus, the MRE solution captures the exact solution for the finite capacity system. Moreover, the MRE solution is of closed form and thus it can be used as a cost effective building block towards the analysis of queuing networks with finite capacity.

Two typical case studies involving the Exponential (M) and Generalized Exponential (GE) interevent-time distributions are applying expressions (14)-(16), (20) and (22) are presented below. 


\subsubsection{M/M/1N Queue}

For a stable $\mathrm{M} / \mathrm{M} / 1 / \mathrm{N}$ queue Lagrangian coefficients $\mathrm{Z}$ and $\mathrm{y}$ are determined by

$$
Z=1-\rho^{N+1}, \quad y=1 .
$$

Thus, the MRE solution for the M/M/1/N queue is identical to the exact state probability

$$
p_{N}(n)=(1-\rho) /\left(1-\rho^{N+1}\right) \rho^{N}, \quad 0 \leq n \leq N .
$$

\subsubsection{GE/GE/1N Queue}

The GE type distribution is of the form

$$
\operatorname{Pr}(W \leq t)=1-\tau \exp (-\sigma t), t \geq 0,
$$

where $\tau=2 /\left(1+C^{2}\right)$ and $\sigma=\tau v$ and $\mathrm{W}$ is the random variable of the interevent time, while $1 / v$ and $\mathrm{C}^{2}$ are the corresponding mean and SCV.

Moreover, the qld of the infinite capacity GE/GE/1 queue is given by [22]

$$
q(n)=p_{\infty}(n)=\left\{\begin{array}{cc}
1-\rho, & n=0, \\
(1-\rho) g x^{n} & n=1,2, \ldots,
\end{array}\right.
$$

For a stable GE/GE/1/N queue $\mathrm{Z}$ and $\mathrm{y}$, can be determined by using constraints (14)-(15) and the prior distribution of the form (26), namely

$$
Z=1-\rho^{2} x^{N-1}, y=(\tau(1-\rho \sigma)+\rho \sigma) /(\tau \sigma),
$$

where $\sigma=2 /\left(1+C a^{2}\right)$ and $\tau=2 /\left(1+C s^{2}\right)$. Thus, the MRE solution for the $\mathrm{GE} / \mathrm{GE} / 1 / \mathrm{N}$ queue is given by

$$
p_{N}(n)=\left\{\begin{array}{lc}
(1 / Z) q(n), & 0 \leq n \leq N-1, \\
(1 / Z) q(n) y, & n=N
\end{array}\right.
$$

and turns out identical to the exact solution for the GE/GE/1/N queue. The latter can be derived directly by carrying out a considerable amount of non-trivial algebraic manipulations to solve the system of GE-type global balance equations via the method of Z-transform.

\section{Internet Link Carrying Realistic TCP Traffic}

The simulation topology used in $n s-2$ [24] to validate the described approach is illustrated in Fig. 1. It assumes that there are 100 source and destination nodes connected via a bottleneck link of capacity 50Mbps and two-way propagation delay of $1 \mathrm{~ms}$. The capacity of the source and destination links equals that of the bottleneck link. The two-way propagation delays of the source and destination links are uniformly distributed in $[2 \mathrm{~ms}, 10 \mathrm{~ms}]$ and $[10 \mathrm{~ms}, 200 \mathrm{~ms}]$, respectively. The buffer at the output queue of S/R 1 towards the bottleneck link has a size of $N$ packets.

The arrival process of new connections is assumed to be Poisson. When a new connection opens it chooses randomly a source and a destination node and performs a data transfer of $S$ packets using the Reno flavor of TCP. The maximum packet size is 1500 bytes and $S$ is exponentially distributed with an average of 30 full-size packets. The maximum window size advertised by the receivers is 32 packets. 


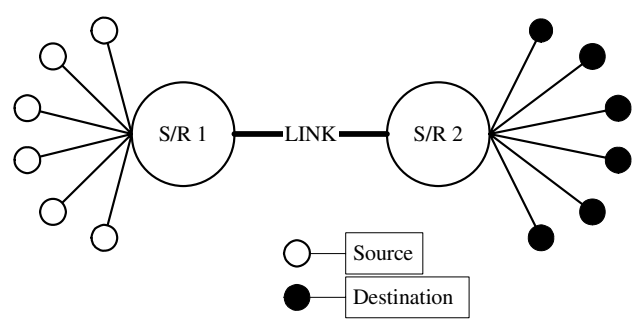

Fig. 1. Internet Link topology

\subsection{ME and MRE Solution for the Internet Link}

In this section the principles of ME and MRE and classical queuing theory are applied to device a computationally efficient solution for the performance analysis of Internet links for the cases of infinite and finite buffer systems.

\subsubsection{ME Solution for the Internet Link with Infinite Buffer}

Let at any given time, $n, n=0,1, \ldots$, be the number of packets in a typical Internet queue as depicted in Fig. 1, $\{q(n)\}$ be the true but unknown steady state probability of having $n$ packets. Suppose all that is known about the state probabilities $q(n)$, $n=0,1, \ldots$, is the following set of mean value constraints:

( i ) Normalization $\sum_{n=0}^{\infty} q(n)=1$,

( ii ) Probability of a busy system $\sum_{n=0}^{\infty} h(n) q(n)=1-q(0)$,

where $h(n)=0, n=0$, or 1 , otherwise,

( iii ) Average queue length, $<n>, \sum_{n=0}^{\infty} n q(n)=<n>$.

Following the reasoning in Section 3, the ME solution for $q(n)$ is given by

$$
q(n)= \begin{cases}1 / Z, & n=0, \\ (1 / Z) g x^{n}, & n>0,\end{cases}
$$

where

$$
1 / Z=q(0), x=1-(1-q(0)) /<n>, g=(1-q(0))(1-x) /(q(0) \cdot x) .
$$

\subsubsection{MRE Solution for the Internet Link with Finite Buffer}

Let at any given time $n(n=0,1, \ldots, N)$ be the number of packets in a typical Internet link queue of finite capacity $N(>0),\{p(n)\}$ be the true but unknown steady state probability of having $n$ packets and $q(n)$ be a prior steady state probability estimate of $p(n)$. Moreover new information takes the form of the following mean value constraints:

(i) Normalization, $\sum_{n=0}^{N} p(n)=1$,

(ii) Full Buffer State Probability, $\{\varphi=p(N), 0<\varphi<1\}, \sum_{n=0}^{N} f(n) p(n)=\varphi$, where $f(n)=1$, if $n=N$, and 0 otherwise. 
In a manner similar to Section 3 the MRE solution for the system is expressed by

$$
p(n)=(1 / Z) q(n) y^{f(n)}, \quad n=0,1, \ldots, N,
$$

where $1 / Z$, and $y$ can be determined by using the set of constraints (34) and (35), and $q(n)$ a the prior estimate for the pdf.

The prior pdf $q(n)$ is estimated by the qld of the corresponding infinite queue. This type of prior is a natural choice which enables the MRE approximation pdf $p(n)$ to capture the exact solution in certain cases (c.f., [23]). In the present analysis the ME solution for the corresponding infinite capacity queue is derived in the previous subsection and proposed as the prior pdf $q(n)$. The form of the MRE solution suggests the proportionality relationships already discussed in Section 4 between the Infinite and Finite Buffer Systems for the case of Internet Links carrying TCP traffic.

\subsection{Validation}

The 'proportionality' relationship between infinite and finite buffer cases is exhibited in Fig. 2 via a set of typical numerical experiments, with system parameters as given in Table 1. Moreover the credibility of the proposed ME (infinite buffer case) and MRE (finite buffer case) solutions towards the analysis of Internet links carrying TCP traffic, outlined in the previous sections, is demonstrated against simulation via the same set of typical numerical experiments.

Table 1. Systems Parameters for the Infinite and Finite Buffer Cases

\begin{tabular}{|c|c|c|c|c|c|c|c|}
\cline { 2 - 8 } \multicolumn{1}{c|}{} & \multicolumn{2}{c|}{ Infinite Buffer System } & \multicolumn{5}{c|}{ Finite Buffer System } \\
\hline$\rho$ & $<n>$ & $q(0)$ & $\mathrm{N}$ & $<n>$ & $p(0)$ & $p(N)$ & $\pi$ \\
\hline 0.3 & 1.029 & 0.864 & 50 & 1.047 & 0.861 & $2.3 \mathrm{E}-4$ & $9.1 \mathrm{E}-4$ \\
\hline 0.6 & 7.347 & 0.550 & 150 & 7.252 & 0.553 & $2.8 \mathrm{E}-5$ & $6.1 \mathrm{E}-5$ \\
\hline 0.8 & 28.368 & 0.268 & 150 & 25.454 & 0.268 & $1.4 \mathrm{E}-3$ & $2.3 \mathrm{E}-3$ \\
\hline 0.9 & 88.998 & 0.098 & 150 & 52.176 & 0.118 & $9.4 \mathrm{E}-3$ & $1.6 \mathrm{E}-2$ \\
\hline
\end{tabular}

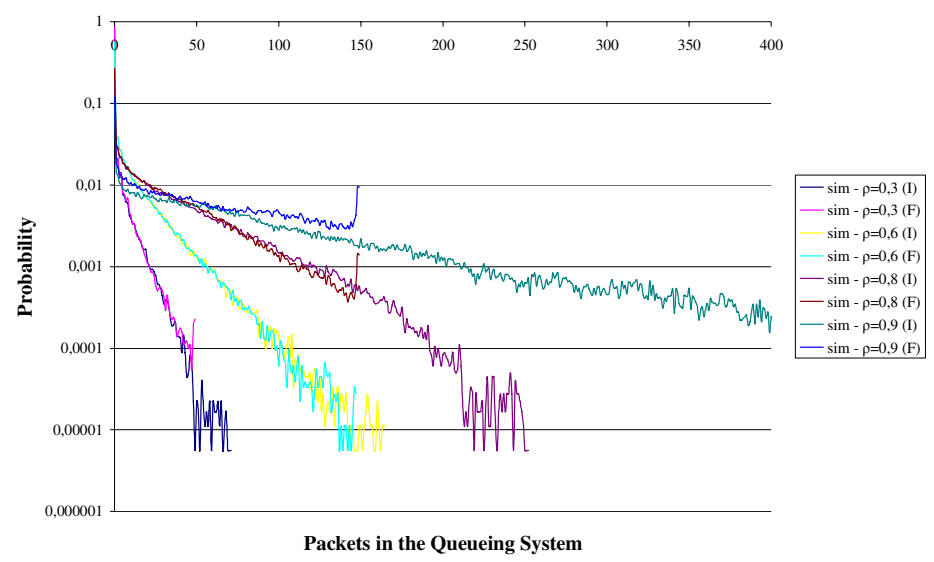

Fig. 2. qlds for the finite (F) and infinite (I) buffer cases obtained from simulation for $\rho=0.3$, $0.6,0.8,0.9$ 


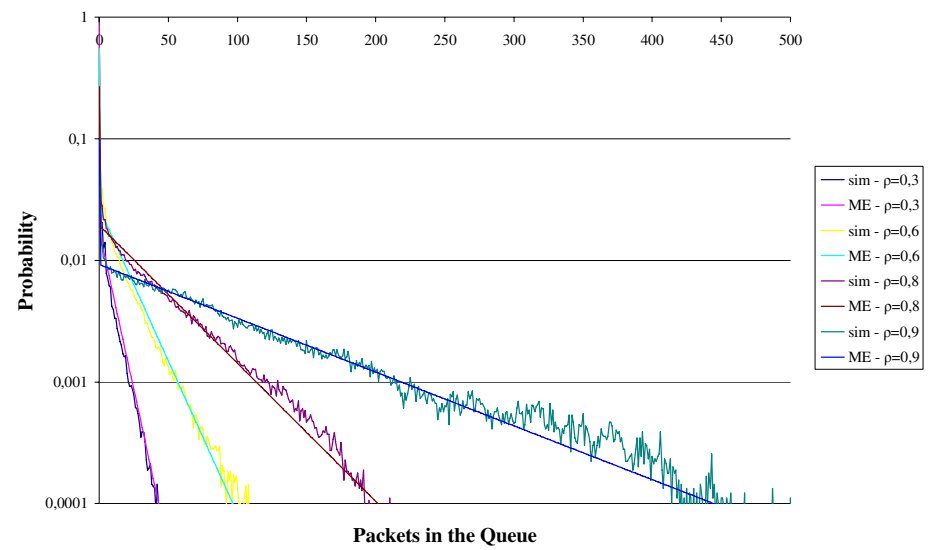

Fig. 3. qlds for the infinite buffer case obtained from simulation and the ME for $\rho=0.3,0.6$, $0.8,0.9$

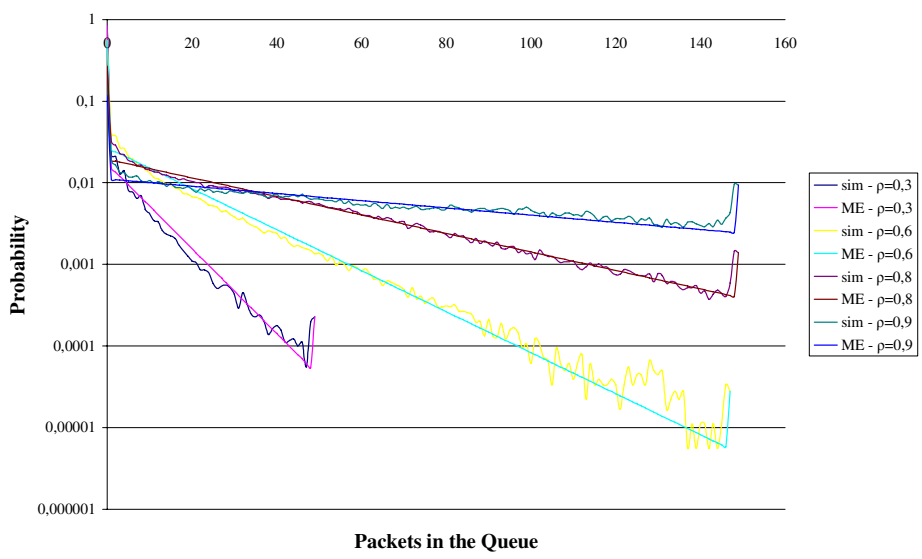

Fig. 4. $q l d \mathrm{~s}$ for the finite buffer case obtained from simulation and the ME for $\rho=0.3,0.6$, $0.8,0.9$

Fig. 3 and Fig. 4 depict the qld for the infinite and finite buffer cases respectively for certain values of utilization, $\rho$. It is apparent, that the suggested approach manage to capture the behaviour of the system under various settings. In the finite buffer case, in particular, the MRE solution produces in accordance to the simulation a certain final peak at the full buffer probability, $p(N)$. An insight on the existence of this final peak is offered by this notion of 'parallel' distributions.

\section{Conclusions}

In this paper the information theoretic principles of Maximum Entropy and Minimum Relative Entropy were applied, as a method of inference towards the analysis of 
infinite and finite capacity systems. In particular, the principle of MRE is applied, as a method of inference, to the general problem of estimating finite buffer stationary qld, given, as a prior estimate, the qld of the corresponding infinite capacity buffer queue. Under the 'proportionality' relationship MRE is shown to provide with exact results for certain typical cases of queuing systems. It is also observed that certain real systems such as Internet links carrying TCP traffic exhibit this 'proportionality' relationship between finite and infinite systems. In the latter case, ME and MRE solutions manage to follow closely the behavior of the real systems, providing this way simple, cost effective, closed form expressions for the queue length distribution of the systems under study. Exhaustive experimentation showed the very good fit of the results with the outcome of simulation runs. Despite its simplicity, the methodology captures the behavior of the system under study both in the cases of finite and infinite buffers, and thus can easily be utilized for network management and design, capacity planning, and congestion control.

Further work is required to identify more subtle constraints that would give exact finite buffer qld for other types of queues with more complex interarrival and service time distributions, to analyze conditions for prescribed degrees of accuracy of approximate MRE solutions applicable to other cases of finite capacity queues where the 'proportionality' condition does not hold, and to extend the study to a network of internet links.

\section{References}

[1] Crovella, M.E., Bestavros, A., "Self-Similarity in World Wide Web Traffic: Evidence and Possible Causes,” In IEEE/ACM Transactions on Networking, 5(6):835-846, (1997).

[2] Grossglauser, M., Bolot, J., "On the Relevance of Long Range Dependence in Network Traffic," In IEEE/ACM Transactions on Networking, 7(5):629-640 (1999).

[3] Feldman, A., Gilbert, A., Huang, P., Willinger, W., "Data networks as cascades: Explaining the multifractal nature of Internet Wan tra.c," In ACM SIGCOMM '98, pp.42-55, Vancouver, Canada (1998).

[4] Willinger, W., Paxson, V., "Where Mathematics meets the Internet," Notices of the American Mathematical Society, 45(8):961-970 (1998).

[5] Erramilli, A., Narayan, O., Willinger, W., "Experimental queuing analysis with longrange dependent packet traffic," In IEEE/ACM Transactions on Networking, 4(2):209223 (1996).

[6] Erramilli, A., Narayan, O., Neidhardt, A., "Performance Impacts of Multi-Scaling in Wide Area TCP/IP Traffic" In IEEE INFOCOM '00, Tel Aviv, Israel (2000).

[7] Ribeiro, V., Riedi, R., Crouse, M., Baraniuk, R., "Multiscale Queuing Analysis of LongRange-Dependent Network Traffic,” In IEEE INFOCOM '00, Tel Aviv, Israel (2000).

[8] Vanichpun, S., Makowski, A., "Positive correlations and buffer occupancy: Lower bound via supermodular ordering," In IEEE INFOCOM '02, New York, NY (2002).

[9] Cardwell, N., Savage, S., Anderson, T., "Modeling TCP Latency," In IEEE INFOCOM '00, Tel Aviv, Israel (2000).

[10] Fredj, S.B., Bonald, T., Proutiere, A., Regnie, G., Roberts, J., "Statistical Bandwidth Sharing: A Study of Congestion at Flow Level," In ACM SIGCOMM '01, pp.111-122, San Diego, USA (2001). 
[11] Barakat, C., Thiran, P., Iannaccone, G., Diot C., Owezarski, P., "A flow-based model for Internet backbone traffic," In ACM Internet Measurement Workshop, Marseille, France (2002).

[12] Garetto, M., Towsley, D., "Modeling, Simulation and Measurements of Queuing Delay under Long-tail Internet Traffic", In SIGMETRICS'03, pp. 47-57, San Diego, USA (2003).

[13] Appenzeller, G., Keslassy, I., McKeown, N., "Sizing router buffers", In ACM SIGCOMM '04, pp. 281-292, USA, August/September 2004.

[14] Benes, V.E., "Mathematical Theory of Connecting Networks and Telephone Traffic", Academic Press, New York (1965).

[15] Ferdinand, A.E., “A Statistical Mechanical Approach to Systems Analysis”, IBM Journal of Research and Development, vol. 14, pp.539-547 (1970).

[16] Pinsky, E., Yemini, Y., "A Statistical Mechanics of Some Interconnection Networks", Performance '84, North-Holland, pp. 147-158 (1984).

[17] Jaynes, E.T., "Information Theory and Statistical Mechanics I", Physical Review, vol.106, pp.620-630 (1957).

[18] Jaynes, E.T., "Information Theory and Statistical Mechanics II", Physical Review, vol.108, pp.171-190 (1957).

[19] Shore, J.E., Johnson, R.W., "Axiomatic Derivation of the Principle of Maximum Entropy and the Principle of Minimum-Cross Entropy", IEEE Trans. on Information Theory, vol.IT-26, pp.26-37 (1980).

[20] Shore, J.E., Johnson, R.W., "Properties of Cross Entropy Minimisation", IEEE Trans. on Information Theory, vol.IT-27, pp.472-482 (1981).

[21] Kouvatsos, D.D., "Maximum Entropy and the G/G/1/N Queue", Acta Informatica, vol.23, pp.545-565 (1986).

[22] Kouvatsos, D.D., “A Maximum Entropy Analysis of the G/G/1 Queue at Equilibrium”, Journal of Oper. Research Society, vol.39, pp.183-200 (1988).

[23] Skianis, C., Kouvatsos, D.D., "Arbitrary Open Queueing Networks with Server Vacation Periods and Blocking", Special Issue on Queueing Networks and Blocking, Annals of Operations Research 79, pp. 143-180 (1998).

[24] McCanne, S., Floyd, S., "Ns-2 network simulator", http://www.isi.edu/nsnam/ns/ 